\title{
Economic evaluation of point-of-care testing in the remote primary health care setting of Australia's Northern Territory
}

This article was published in the following Dove Press journal:

ClinicoEconomics and Outcomes Research

\section{Brooke A Spaeth' \\ Billingsley Kaambwa ${ }^{2}$ \\ Mark DS Shephard' \\ Rodney Omond ${ }^{3}$}

'Flinders University International Centre for Point-of-Care Testing, Flinders University, Adelaide, SA, Australia; ${ }^{2} \mathrm{Health}$ Economics Unit, College of Medicine and Public Health, Flinders University, Adelaide, SA, Australia; ${ }^{3}$ Primary Health Care Branch, Top End Health Service, Department of Health, Northern Territory Government, Darwin, NT, Australia
Correspondence: Brooke A Spaeth Flinders University International Centre for Point-of-Care Testing, Level 3 West Wing, Sturt Campus, Flinders University, Bedford Park, SA 5042, Australia

Tel $+6 \mid 8820 I 7555$

Fax +61882017666

Email brooke.spaeth@flinders.edu.au
Aim: To determine the cost-effectiveness of utilizing point-of-care testing (POCT) on the Abbott i-STAT device as a support tool to aid decisions regarding the emergency medical retrievals of patients at remote health centers in the Northern Territory (NT) of Australia.

Methods: A decision analytic simulation model-based economic evaluation was conducted using data from patients presenting with three common acute conditions (chest pain, chronic renal failure due to missed dialysis session(s), and acute diarrhea) at six remote NT health centers from July to December 2015. The specific outcomes measured in this study were the number of unnecessary emergency medical retrieval prevented through POCT. Cost savings through prevented unnecessary medical retrievals for each presentation type were then determined and extrapolated to give per annum NT-wide estimates.

Results: POCT prevented 60 unnecessary medical evacuations from a total of 200 patient cases meeting the selection criteria (48/147 for chest pain, 10/28 for missed dialysis, and 2/25 for acute diarrhea). The associated cost savings were AUD \$4,674, \$8,034, and \$786 per patient translating to NT-wide savings of AUD \$13.72 million, \$6.45 million, and \$1.57 million per annum (AUD \$21.75 million in total) for chest pain, missed dialysis, and acute diarrhea presentations, respectively.

Conclusion: This study demonstrated that POCT when used to aid decision making for acutely ill patients delivered significant cost savings for the NT health care system by preventing unnecessary emergency medical retrievals.

Keywords: acute, remote health, primary care, retrieval, acute care, myocardial infarction, dialysis, dehydration, indigenous health, pathology testing, medical retrieval, cost-effectiveness

\section{Introduction}

The health status of Australians living in rural and remote communities is generally poorer than that of matched populations living in urban Australia. This trend is consistent across both chronic and acute disease presentations. ${ }^{1}$ Remotely located patients requiring urgent medical care are most often transferred through aerial medical retrieval services to the nearest metropolitan hospital emergency department. The decision to evacuate a patient from a remote health facility is made based on the best available evidence at the time using clinical interpretation, basic medical equipment, and discussion with an on-call medical practitioner often based at a tertiary hospital. The lack of medical staff and equipment often necessitates that the patient is evacuated so as to err on the side of caution. This is because in the remote Northern Territory (NT), if patients are not able to remain in community because of clinical requirements, 
they must be evacuated as no overnight clinical services are available within these remote health facilities. This may lead to unnecessary hospitalizations. The Australian Institute of Health and Welfare reported that across 2008-2009, 8.5\% of all hospitalizations (30.6/1000 persons) in Australia could have been avoided if managed effectively out of hospital. ${ }^{2}$ This trend is amplified with increasing remoteness for most acute and chronic conditions with the NT having the highest rate of avoidable hospitalizations in Australia (47.8/1000 persons). ${ }^{2}$

In the NT, the cost of aeromedical evacuations is borne by the NT Department of Health, which quotes a cost of $\$ 141.59$ per minute flight time in its Fees and Charges Manual. ${ }^{3}$ For a 1 -hour return medical retrieval, this equates to a total cost of $\$ 8,495$ per evacuation. This figure is consistent with published data from rural and remote Queensland aeromedical evacuations of $\$ 8,520$ per 1-hour interhospital transfer. ${ }^{4}$ The cost of medical evacuations via helicopter is substantially higher at $\$ 16,171$ per 1 -hour return flight. ${ }^{3}$ The CareFlight 2014/15 Annual Report documented a total of 2789 emergency evacuations for the Top End of the NT (111 [4\%] of which were via helicopter), ${ }^{5}$ based on an average 2-hour return flight; this equates to an annual cost estimate of $\$ 47$ million dollars per annum for the Top End jurisdiction alone. Rural and remote disadvantage is also reflected in poorer standards of general services and health infrastructure including pathology services. Point-of-care testing (POCT) allows pathology testing to be conducted during a patient visit with results immediately available for patient care. ${ }^{6}$ POCT has a particular niche in rural and remote communities where access to mainstream laboratory services is generally poor, there are long delays in transporting pathology samples to laboratories, turnaround time for delivery of test results back to the local health service may be slow, and patient loss to follow-up is high. ${ }^{7,8}$ For medical emergencies in these sectors, the speed of POCT provides critical practical and operational benefits by providing another tool to assist in the triage of acutely ill patients. The additional clinical information provided by POCT adds valuable data within the patient assessment. These extra data reduce the intrinsic risk implied by deciding to leave a patient in a community, with its lower level of available clinical care. POCT also identifies patients at high risk but an absence of clinical signs (eg, a patient clinically stable post infarct).

POCT is considered to be generally more expensive than traditional laboratory pathology testing as it is not able to achieve the same economy of scale. ${ }^{9-11}$ However, a limited number of studies have examined the cost effectiveness of
POCT in a rural or remote context. One previous study based on data from a hospital in rural New Zealand demonstrated that POCT improved diagnostic certainty and thereby reduced the number of transfers to the major base hospital by $62 \%$ and increased weekly discharges from seven pre-POCT to 34 post-POCT, with associated cost savings of NZ\$362,138 per annum. ${ }^{12}$

Previous studies from our research group have evaluated the analytical safety, operational efficiency, and clinical effectiveness of the i-STAT (Abbott Point of Care, Princeton, NJ, USA) POCT device in the primary care setting in remote NT. ${ }^{13-16}$ The use of the i-STAT device in the remote NT includes tests for cardiac troponin I, electrolytes, blood gases, urea, creatinine, glucose, ionized calcium, and international normalized ratio (INR) with results for each test available in 10 minutes or less. The present study from our group is the first to provide an economic evaluation of POCT on the i-STAT for acute presentations in this setting. Our hypothesis is that POCT will prevent unnecessary medical evacuations and thereby lead to cost savings. Unnecessary evacuations were defined as the number of evacuations that would have taken place in the absence of POCT results, that is, based solely on clinical interpretation, basic medical equipment, and discussion with an on-call off-site medical practitioner. This analysis took the form of a model-based probabilistic economic evaluation assessing whether POCT leads to costs savings through preventing unnecessary medical retrievals when compared to usual care.

\section{Methods}

\section{Site selection}

In Australia's NT, remote health care is provided through primary health care facilities that have limited infrastructure, resources, and staffing levels, with high rates of staff turnover. These remote health facilities are generally located many hundreds of kilometers from the nearest major tertiary hospital, necessitating air transport as the best (and often only) option for acutely ill patients.

Six remote health services in the NT that have access to on-site POCT (and were current participants in the NT i-STAT POCT Program) were selected to evaluate the clinical and cost effectiveness of the management of acute patient presentations. The six remote health centers comprised two large centers (servicing an indigenous population base of between 2000 and 3000 clients), two medium-sized centers ( $\sim 1000$ clients), and two small centers ( $<500$ clients). Two of the health centers were located in the Central Australian region (which covers the southern half of the NT which 
comprises a mainly desert environment) and four were from the Top End of the NT (which comprised the tropical northern half of the Territory).

\section{Acute presentations}

The economic evaluation in this study focused on three common acute presentations observed in the NT: chest pain or other symptoms suggestive of acute coronary syndrome (ACS) with no obvious ST-elevation on electrocardiogram (ECG); symptoms resulting from missed dialysis session(s) in the setting of chronic renal failure (CRF); and symptoms suggestive of acute dehydration due to diarrhea and/or vomiting. A detailed description of the normal pathways for each acute condition and how POCT changes these pathways was provided in a separate paper by our research group, which outlined the clinical effectiveness of POCT. ${ }^{15}$ The i-STAT tests investigated in this study were troponin I for ruling out ACS; potassium and creatinine to measure CRF patient risk; and sodium, potassium, and chloride for assessing possible acute dehydration.

\section{Adverse events}

To determine if patients who remained in the remote communities experienced any adverse events, the electronic case notes within the Patient Care Information System (PCIS) were examined for each patient 10 days after their initial presentation. Adverse events included any secondary acute presentation, a medical evacuation, or death.

\section{Ethics registration}

Data on the prevalence of the three presentation types in patients serviced by the remote health center network of the NT Department of Health were sourced from the NT Health Data Warehouse (NTHDW), requiring the investigators to gain ethics approval for this project from the Menzies School of Health Research Ethics Committee (Application Number 2015-2469, approved September 2015). A data release application form for NTHDW was also lodged in November 2015 and approved on May 20, 2016. A condition of ethics approval was that all remote community and individual patient identities must be kept anonymous.

\section{Development of the economic evaluation model}

A decision analytic simulation model (DASM) or "Decision Tree" 17,18 was used to assess whether POCT leads to cost savings compared to usual care in the three separate acute medical presentations outlined above. A modeling framework is ideally suited to demonstrate and explore the importance of the inherent uncertainty in the cost-effectiveness question. ${ }^{19}$ In the POCT arm, the decision to medically evacuate a patient was made on the basis of on-site i-STAT results from the participating remote health centers. In the usual care arm, the project's Chief Clinical Investigator (Senior Rural Medical Practitioner in the NT) provided an independent clinical judgment on whether each patient would or would not have been evacuated to hospital should the results from the i-STAT device not have been available at the time of the presentation. This decision was based on clinical interpretation, results of basic medical equipment tests, and review of the patient's electronic record. This process is exactly the way clinical decisions about evacuations were made routinely prior to POCT being available at all sites, with the Clinical Advisor having considerable experience in these decisionmaking processes, both before and after the introduction of POCT in the NT.

\section{Model structures and inputs}

The structures of the DASM for each condition are shown in Figure 1. The pathways for the modeled acute presentations within the POCT and usual care arms were designed to mirror those observed in the general population during the course of this study. The pathways were also informed by a review of the literature and advice from clinical experts on the research team. The model combined estimates of probabilities relating to transitions between the models' health states, resource use/ cost, and the number of evacuations avoided in each arm.

Probabilities providing a quantitative estimate of the likelihood that a given event within the clinical pathways would occur were obtained from the NT Department of Health PCIS and are presented for both POCT and usual care arms (data supplied in Table S1).

Resource use and subsequent costs associated with both the POCT and usual care arms were estimated to calculate mean costs per patient for both arms (Table S2). Total costs per patient were estimated by combining resource use data and unit costs for these resources (derived from published data sets including those from the NTHDW). Total costs per patient were calculated as the sum of staff costs (incurred when carrying out assessments and tests and undergoing training), equipment costs (including costs of the i-STAT device), and costs of supplies (eg, consumables such as i-STAT cartridges, syringes for blood sampling, gloves, and protective equipment). Drug costs (eg, morphine, clopidogrel, enoxaparin, calcium resonium, and lactulose) and treatment costs (eg, oxygen, saline, and oral hydration solutions), other 

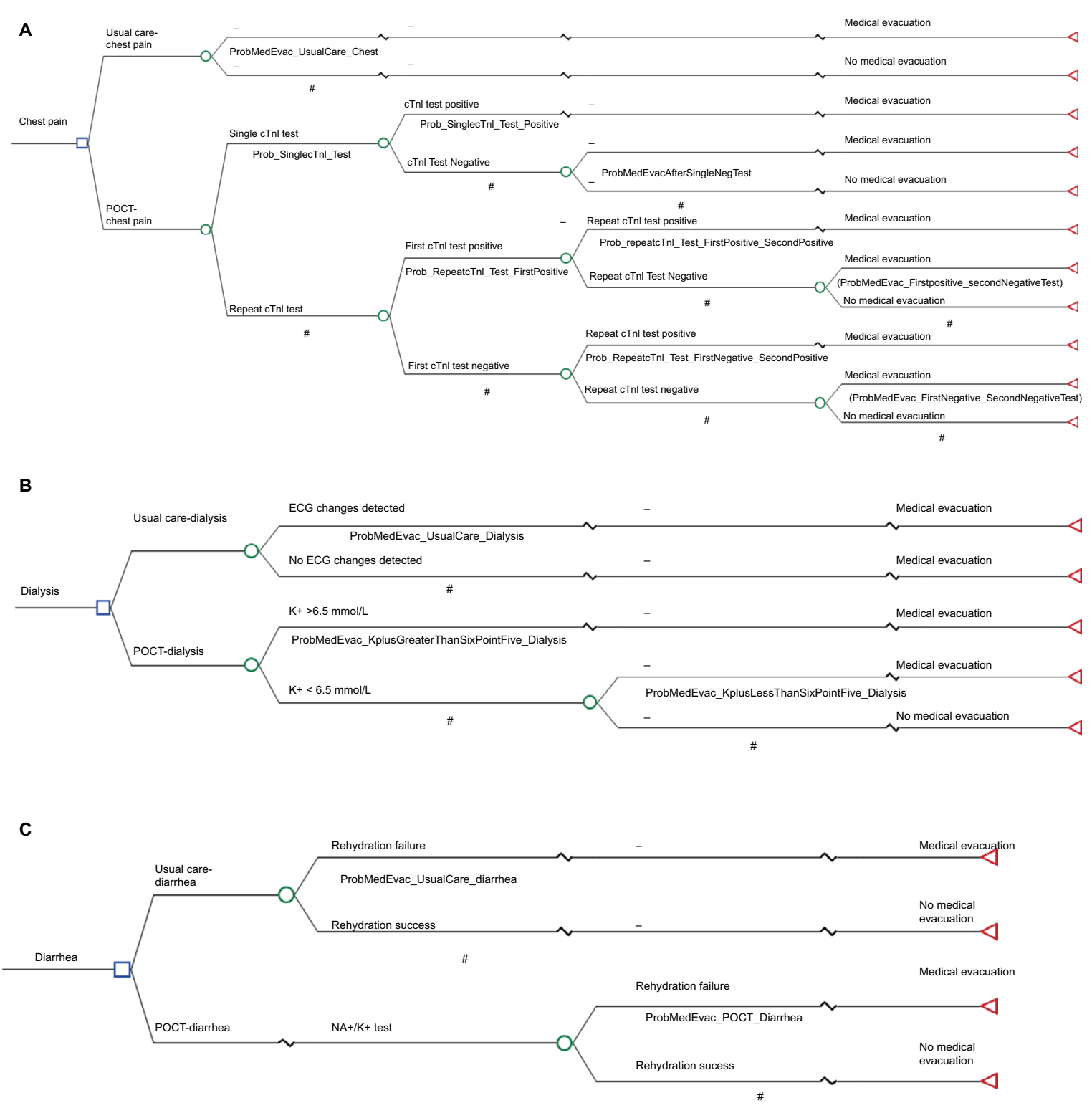

Figure I Decision tree models depicting clinical pathways for "Point-of-Care Testing" compared to "Usual Care" for the three medical presentations.

Notes: (A) Patients with acute chest pain without ST elevation on ECG. (B) Patients with chronic renal failure who missed a dialysis session(s). (C) Patients with acute diarrhea and evidence of dehydration. In all models, "\#" is I - the probability in the branch above; Medical evacuation, individual was medically evacuated; No medical evacuation, individual was not medically evacuated. Model A: Usual Care - Chest Pain, usual care arm for individuals with acute chest pain; ProbMedEvac_UsualCare_Chest, probability of being evacuated in the usual care arm; POCT - Chest Pain, point of care test arm for individuals with acute chest pain; Single cTnl test, pathway for individuals that have a single cardiac troponin I test within the POCT arm; Prob_SinglecTnl_test_Positive, probability of having a positive result from a single cTnl test; cTnl test positive, having a positive result from a single cTnl test; Prob_SinglecTnl_test_Positive, probability of having a positive result from a single cTnl test; cTnl test negative, having a negative result from a single cTnl test; ProbMedEvacAfterSingleNegTest, probability of being evacuated following a negative result from a single cTnl test; Repeat cTnl test, pathway for individuals that have a repeat cTnl test within the POCT arm; First cTnl test positive; having a positive result from the first of repeat cTnl test; Prob_RepeatcTnl_ test_FirstPositive, probability of having a positive result from the first of repeat cTnl tests; Repeat cTnl test positive, having a positive result on the second cTnl test; Prob_RepeatcTnl_test_FirstPositive_SecondPositive, probability of having two positive results from the repeat cTnl tests; Repeat cTnl test negative, having a negative result on the second cTnl test; ProbMedEvac_FirstPositive_SecondNegativeTest, probability of being evacuated following a positive and then a negative result from repeat cTnl tests; First cTnl test negative, having a negative result from the first of repeat cTnl test; Prob_RepeatcTnl_test_FirstNegative_SecondPositive, probability of having negative and then a positive result from the repeat cTnl tests; ProbMedEvac_FirstNegative_SecondNegativeTest, probability of being evacuated following two negative results from repeat cTnl tests. Model B: Usual Care - Dialysis, usual care arm for individuals with chronic renal failure who missed a dialysis session(s); ECG changes detected, pathway for individuals in whom ECG changes were detected; ProbMedEvac_UsualCare_Dialysis, probability of being evacuated following ECG changes being detected; No ECG changes detected, pathway for individuals in whom no ECG changes were detected; POCT - Dialysis, point of care test arm for individuals with chronic renal failure who missed a dialysis session(s); $\mathrm{K}+>6.5 \mathrm{mmol} / \mathrm{L}$, pathway for individuals who had a positive potassium assay test result; ProbMedEvac_KplusGreaterThanSixPointFive_Dialysis, probability of being evacuated following a positive potassium assay test result; $\mathrm{K}+<6.5 \mathrm{mmol} / \mathrm{L}$, pathway for individuals who had a negative potassium assay test result; ProbMedEvac_ KplusLessThanSixPointFive_Dialysis, probability of being evacuated following a negative potassium assay test result. Model C: Usual Care - diarrhea, usual care arm for individuals with acute diarrhea and evidence of dehydration; Rehydration failure, pathway for individuals who had rehydration failure; ProbMedEvac_UsualCare_Diarrhea, probability of having rehydration failure in the usual care arm which is equal to the probability of being evacuated following rehydration failure in this arm; Rehydration success, pathway for individuals who had rehydration success; POCT - Diarrhea, point of care test arm for individuals with acute diarrhea and evidence of dehydration; $\mathrm{Na}+$ / $\mathrm{K}+$ test, sodium and potassium test used in the POCT arm; ProbMedEvac_UsualCare_Diarrhea, probability of having rehydration failure in the POCT arm which is equal to the probability of being evacuated following rehydration failure in this arm.

Abbreviations: cTnl, cardiac troponin I; ECG, electrocardiogram; POCT, point-of-care testing. 
ongoing operational costs of conducting i-STAT testing and other clinical assessments, and costs of medical evacuations also formed part of the total costs. Equipment and training costs were annuitized at $5 \%$ and based on a conservative lifetime of 5 years. ${ }^{20}$ An annual maintenance cost for equipment of $\$ 1,039$ was also included in the costing. All resource costs used in the model are reported in Australian dollars at 2017/18 unit prices. The Supplementary materials provide a description of the resource use and data estimates split into pre-evacuation, evacuation, and prevalence estimates.

\section{Economic evaluation}

A patient-level analysis was undertaken from a health sector (Medicare) cost perspective and the results reported in terms of cost savings because of prevention of unnecessary medical evacuation. Probabilistic analyses were used in the base case and sensitivity analyses based on 100,000 Monte Carlo simulations. To facilitate the probabilistic analyses, beta distributions were used to model the probability of transitions between health states while gamma distributions were fitted to all costs.

The availability of estimates on the cost of a medical evacuation for a single patient (Table S3) enabled incremental cost savings due to medical evacuations avoided to be calculated. These savings were calculated as the average weighted round-trip cost of a medical evacuation times the number of unnecessary medical evacuations avoided less the incremental cost (Table S3 $)$. Per patient incremental cost savings for each medical presentation were then extrapolated to the general NT population through multiplying them by the prevalence estimates for each respective medical presentation.

Sensitivity analysis for the economic evaluation was undertaken to assess uncertainty in the cost savings due to avoided medical evacuations by splitting the sample into Top End services ( $\mathrm{n}=40$ services) cost of per medical evacuation or Central Australian services ( $n=32$ services) and applying the respective costs of medical evacuations for these services (Table S3).

\section{Results}

A summary of the economic evaluation results provided in Table 1 shows that the mean \pm standard error costs per patient (presented for the usual care and POCT arms, respectively) were $\$ 257 \pm 1$ and $\$ 341 \pm 1$ (acute chest pain), $\$ 274 \pm 1$ and $\$ 308 \pm 1$ (missed dialysis), and $\$ 204 \pm 1$ and $\$ 306 \pm 1$ (acute diarrhea). The total cost of a round-trip medical evacuation was estimated as $\$ 22,560$ per patient (data supplied in Table S3).

\section{Patients presenting with chest pain (without ST elevation on ECG)}

Compared to usual care, POCT for patients with acute chest pain (POCT - Chest Pain) was more expensive (by $\$ 84$ per patient; 95\% confidence interval [CI]: \$81-\$86) but also more effective (prevented 0.2109 unnecessary medical evacuations per patient; 95\% CI: 0.2106-0.2112, Table 1). Adopting the "POCT - Chest Pain" strategy and then spend-

Table I Costs, effectiveness, and cost savings based on the number of unnecessary medical evacuations avoided

\begin{tabular}{|c|c|c|c|c|c|c|c|}
\hline Strategy & $\begin{array}{l}\text { Mean (SE) } \\
\text { cost per } \\
\text { patient (AU \$) }\end{array}$ & $\begin{array}{l}\text { Difference }(95 \% \mathrm{CI}) \\
\text { in costs }(A \cup \$)\end{array}$ & $\begin{array}{l}\text { Mean (SE) } \\
\text { effectiveness }^{\mathbf{a}} \\
\text { per patient }\end{array}$ & $\begin{array}{l}\text { Difference } \\
(95 \% \mathrm{Cl}) \text { in } \\
\text { effectiveness }^{\mathrm{a}}\end{array}$ & $\begin{array}{l}\text { Cost savings } \\
\text { per patient }{ }^{\mathrm{b}}- \\
\text { NT }(A \cup \$)\end{array}$ & $\begin{array}{l}\text { Cost savings } \\
\text { per patient } \\
\text { TE }(A \cup \$)\end{array}$ & $\begin{array}{l}\text { Cost savings } \\
\text { per patient } t^{\mathrm{b}}- \\
\text { CA }(A \cup \$)\end{array}$ \\
\hline \multicolumn{8}{|c|}{ Acute chest pain } \\
\hline Usual care & $257.20(0.85)$ & $83.76(8 I .4 I-86.12)$ & $0.64613(0.0012)$ & $\begin{array}{l}0.21090 \\
(0.21063-0.21117)\end{array}$ & $4,674.11$ & $5,292.36$ & $3,630.18$ \\
\hline РОСТ & $340.97(0.85)$ & & $0.85703(0.00010)$ & & & & \\
\hline \multicolumn{8}{|c|}{ Missed dialysis } \\
\hline Usual care & $273.70(0.77)$ & $34.38(32.21-36.45)$ & $0.03557(0.00012)$ & $\begin{array}{l}0.35769 \\
(0.35721-0.35817)\end{array}$ & $8,034.96$ & $9,083.52$ & $6,264.46$ \\
\hline РOCT & $308.08(0.61)$ & & $0.39326(0.00031)$ & & & & \\
\hline \multicolumn{8}{|c|}{ Acute diarrhea } \\
\hline Usual care & $204.38(0.53)$ & 101.58 (99.91-103.25) & $0.56059(0.00034)$ & $\begin{array}{l}0.03934 \\
(0.03835-0.04033)\end{array}$ & 785.93 & 901.26 & 591.20 \\
\hline POCT & $305.96(0.59)$ & & $0.59993(0.00030)$ & & & & \\
\hline
\end{tabular}

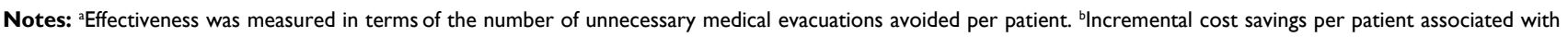
POCT because of medical evacuations avoided were calculated as the average weighted round-trip cost of a medical evacuation ( $\$ 21,717$ [base case - NT as a whole], $\$ 24,539$ $[T E]$, and $\$ 16,952[C A]$ ) times the difference in the number of medical evacuations avoided (ie, incremental effectiveness) less the incremental cost.

Abbreviations: AU, Australian Dollars; CA, Central Australia; Cl, confidence interval; NT, Northern Territory; POCT, point-of-care testing; SE, standard error; TE, Top End. 
Table 2 Total cost savings estimates for the Northern Territory, Top End, and Central Australia

\begin{tabular}{|c|c|c|c|c|c|c|}
\hline Acute presentation type & $\begin{array}{l}\text { POCT cost } \\
\text { savings per } \\
\text { patient (AU \$) }\end{array}$ & $\begin{array}{l}\text { PCIS prevalence } \\
(\%)^{\mathrm{a}}\end{array}$ & $\begin{array}{l}\text { Estimated } \\
\text { remaining } \\
\text { prevalence }^{\text {b }}\end{array}$ & $\begin{array}{l}\text { NT total } \\
\text { estimated } \\
\text { prevalence }^{c}\end{array}$ & $\begin{array}{l}\text { Average cost } \\
\text { of evacuation } \\
(A U \$)\end{array}$ & $\begin{array}{l}\text { Total cost } \\
\text { savings (AU } \\
\$ \text { millions) }\end{array}$ \\
\hline Chest pain without ST-elevation on & $\$ 4,674$ & $|62|(6.98)$ & 1315 & 2936 & $\$ 22,560$ & $\$ 13.72$ \\
\hline \multicolumn{7}{|l|}{ ECG } \\
\hline Top End & $\$ 5,292$ & $1239(7.55)^{d}$ & $934^{\mathrm{e}}$ & 2173 & $\$ 25,49 \mathrm{I}^{\mathrm{f}}$ & $\$ 11.50$ \\
\hline Central Australia & $\$ 3,630$ & $382(5.62)^{g}$ & $38 \mathrm{I}^{\mathrm{h}}$ & 763 & $\$ 17,610$ & $\$ 2.77$ \\
\hline Chronic renal failure with missed & $\$ 8,035$ & $427(1.84)$ & $352(1.84)$ & 803 & $\$ 22,560$ & $\$ 6.45$ \\
\hline \multicolumn{7}{|l|}{ dialysis session } \\
\hline Top End & $\$ 9,084$ & $204(1.24)^{d}$ & $154(1.24 \%)^{\mathrm{e}}$ & 358 & $\$ 25,49 \mathrm{I}^{\mathrm{f}}$ & $\$ 3.24$ \\
\hline Central Australia & $\$ 6,264$ & $223(3.38)^{g}$ & $222(3.38 \%)^{\mathrm{h}}$ & 445 & $\$ 17,610$ & $\$ 2.79$ \\
\hline $\begin{array}{l}\text { Acute diarrhea with symptoms of } \\
\text { dehydration }\end{array}$ & $\$ 786$ & $1097(4.73)$ & $906(4.73 \%)$ & 2001 & $\$ 22,560$ & $\$ 1.57$ \\
\hline Top End & $\$ 901$ & $78 I(4.76)^{d}$ & $589(4.76 \%)^{\mathrm{e}}$ & 1370 & $\$ 25,49 \mathrm{I}^{\mathrm{f}}$ & $\$ 1.23$ \\
\hline Central Australia & $\$ 591$ & $316(4.65)^{g}$ & $315(4.65 \%)^{\mathrm{h}}$ & 631 & $\$ 17,610$ & $\$ 0.37$ \\
\hline Top End total savings & & & & & & $\$ 15.98$ \\
\hline Central Australia total savings & & & & & & $\$ 5.84$ \\
\hline Northern Territory total savings & & & & & & $\$ 21.75$ \\
\hline
\end{tabular}

Notes: ${ }^{a} \mathrm{PCIS}$ prevalence $=$ direct prevalence of each condition obtained from 2015 NT Data Warehouse figures (ie, number of patients with the condition/total number of people living in remote communities serviced by Department of Health remote health centers in the NT). ${ }^{21}{ }^{b}$ Estimated remaining prevalence $=\%$ prevalence from Data Warehouse figures multiplied by the total number of people living in remote communities serviced by the Aboriginal community-controlled health sector in the NT; ${ }^{21}$ it assumes that \% prevalence from Data Warehouse figures is the same for remote communities serviced by the Aboriginal community-controlled health sector. ${ }^{\mathrm{C}} \mathrm{NT}$ total estimated prevalence=sum of $\mathrm{a}+\mathrm{b}$. ${ }^{\mathrm{d}} \mathrm{Top}$ End PCIS population=16402; ${ }^{\mathrm{T}} \mathrm{Top}$ End NGO population=I2372; $\mathrm{f}_{\text {More }}$ weight was given to three large sites/centers in the Top End as determined by the number of evacuations in this calculation. ${ }^{8} \mathrm{CA}$ PCIS population=6794; Total PCIS population=23I96. ${ }^{\mathrm{h}} \mathrm{CA}$ NGO population=6782; Total NGO population=19154. NT total remote population=42,350.

Abbreviations: AU, Australian Dollars; ECG, electrocardiogram; NT, Northern Territory; PCIS, Patient Care Information System; POCT, point-of-care testing.

ing $\$ 34,097$ per 100 patients, as opposed to $\$ 25,720$ per 100 patients under the "Usual Care - Chest Pain" strategy, would lead to 21 unnecessary medical evacuations being avoided (Table 1). Adopting the "POCT - Chest Pain" strategy in place of usual care would lead to cost savings (due to unnecessary medical evacuations avoided) of $\$ 4,674$ per patient, translating to cost savings of $\$ 13.72$ million per annum for the entire NT population (Table 2) based on an acute chest pain prevalence figure estimate of 2,936 for the NT in 2015.

\section{Patients presenting with CRF/missed dialysis session(s)}

POCT for patients with CRF who missed one or more dialysis sessions (POCT - Missed Dialysis) was more expensive (by $\$ 34$ per patient; $95 \%$ CI: $\$ 32$ to $\$ 36$ ) than usual care for these patients (Usual Care - Missed Dialysis) but also more effective (prevented 0.3577 unnecessary medical evacuations per patient; 95\% CI: 0.3572-0.3582). Adopting the "POCT Missed Dialysis" strategy and then spending $\$ 30,808$ per 100 patients, as opposed to $\$ 27,370$ per 100 patients under the "Usual Care - Missed Dialysis" strategy, would lead to 36 unnecessary medical evacuations being avoided (Table 1). A decision to adopt POCT instead of usual care for patients who missed dialysis would lead to cost savings of $\$ 8,035$ per patient, translating to cost savings of $\$ 6.45$ million per annum for the entire NT population (Table 2) based on a
2015 prevalence figure estimate of 803 people that missed dialysis in the NT in 2015.

\section{Patients presenting with acute diarrhea}

POCT for patients with acute diarrhea (POCT - Diarrhea) was more expensive than usual care for these patients (Usual Care - Diarrhea) by $\$ 102$ per patient (95\% CI: $\$ 100-\$ 103$ ) but also more effective (ie, this strategy prevented 0.04 unnecessary medical evacuations per patient; 95\% CI: 0.0384-0.0403). Adopting the "POCT - Diarrhea" strategy and then spending $\$ 30,596$ per 100 patients, as opposed to $\$ 20,438$ per 100 patients under the "Usual Care - Diarrhea" strategy, would lead to four unnecessary medical evacuations being prevented (Table 1). Adopting the "POCT - Diarrhea" strategy in place of usual care would lead to cost savings of $\$ 786$ per patient, translating to cost savings of $\$ 1.57$ million per annum for the entire NT population (Table 2) based on an acute diarrhea prevalence figure estimate of 2,001 for the NT in 2015.

\section{Sensitivity analysis}

In the sensitivity analyses, restricting the analysis to just the Top End services ( $\mathrm{n}=40$; cost per medical evacuation per patient $=\$ 25,491$ as per Table $\mathrm{S} 3$ ) resulted in cost savings of $\$ 5,292$ per patient for acute chest pain, $\$ 9,084$ per patient for missed dialysis, and $\$ 901$ per patient for acute diarrhea. The corresponding overall savings for these Top End services 
were \$1 1.50 million, \$3.24 million, and \$1.23 million per annum (Table 2). When the analysis focussed on Central Australian services $(n=32$; cost per medical evacuation per patient $=\$ 17,610$ - as per Table $\$ 3$ ), cost savings per patient were $\$ 3,630$ (acute chest pain), \$6,264 (missed dialysis), and $\$ 591$ (acute diarrhea) leading to corresponding overall savings for these services of $\$ 2.77$ million, $\$ 2.79$ million, and \$0.37 million per annum, respectively.

No adverse events were recorded within 10 days of the initial presentation for patients who remained in the community.

\section{Discussion}

A recent international study highlighted the importance of conducting setting-specific cost-effectiveness studies for POCT and the need for clinicians, policymakers, and industry to address the gaps in knowledge base in this area. ${ }^{22}$ This study is the first in Australia to provide a detailed economic evaluation of acute POCT in the remote primary care setting.

Within this setting, POCT was shown to deliver an estimated cost saving of $\$ 21.75$ million because of prevention of unnecessary medical retrievals for the whole NT. In terms of clinical presentation, savings were greatest in the "chest pain" patient group ( $\$ 13.72$ million) compared to the savings in the "missed dialysis" (\$6.45 million) and the "acute diarrhea" groups ( $\$ 1.57$ million). The differences in costs savings for Central Australia (\$5.94 million) and Top End (\$15.98 million) were due to differences in prevalence and total numbers of evacuations in these jurisdictions. Although the cost of delivering the POCT pathway per patient was slightly higher (by $\$ 84, \$ 34$, and $\$ 102$, respectively) for the three clinical presentations than the usual care pathway, the cost savings in prevented evacuations far outweighed this small cost impost.

This research highlights that POCT can produce substantial cost savings, which significantly outweigh operational costs, through preventing unnecessary medical evacuations when used in the rural and remote health care setting, where access to timely pathology results is not available. These cost savings could be realized by other rural and remote jurisdictions nationally and internationally through adoption of quality-assured POCT networks to aid decision making for acute presentations with the additional benefit of increasing patient safety.

This study has some limitations. First, this research project did not investigate the additional in-hospital costs saved through preventing admission to a tertiary hospital. The effects of unnecessary medical evacuations on patient/ community (eg, loss of productivity or social or emotional wellbeing of Indigenous patients) were also not included in this study. However, an Australian report on the economic value of pathology found that troponin testing alone reduced the number of admissions to metropolitan emergency departments resulting in cost savings of $\sim \$ 166.5$ million per annum. ${ }^{23}$ The same report also estimated that troponin testing enabled an early discharge strategy to be safely pursued for $\sim 40 \%$ of patients with suspected ACS. ${ }^{23}$ This finding is similar to the present study in which $38 \%$ of patients presenting with chest pain (and no ST-elevation) did not require an evacuation as a result of on-site troponin I testing on the i-STAT device. Therefore, including data on admissions prevented as well as broader costs and outcomes would most likely have made POCT even more cost-effective. Costs associated with keeping the patient in a remote health facility for monitoring were also not included in this study. These costs are, however, estimated to be minimal as no overnight stay beds are available in remote health clinics because of NT government policy.

Second, the clinical and cost effectiveness of POCT in this study was only examined for three common acute presentation types. A survey of clinical staff indicated that the i-STAT was also being used for additional acute presentations such as respiratory disorders and sepsis, as well as for the management of patients on anticoagulation therapy. The use of the i-STAT for these presentations is likely to provide additional clinical and cost benefits, which have not been part of this study. It is also noteworthy that the cost of administering the entire NT POCT Program, operational costs, and the cost of the i-STAT device (including servicing and maintenance) were calculated for all POCT conducted on the i-STAT device during the study period (including for example INR, blood gas, and lactate testing). A small percentage of evacuations in the NT are provided by helicopter $(<5 \%)$; this mode of evacuation is extremely costly ( $\$ 16,167$ per hour compared to $\$ 8,495$ per hour for a fixedwing air evacuation) but was not factored into the economic evaluation because data on the number of helicopter evacuations were not able to be sourced from the Central Australian region; as a result, the total cost savings documented in this study may be slightly underestimated. The average per patient cost of using the i-STAT was calculated to be $\$ 58$; however, the costs per patient varies depending on the number of patient tests performed on the i-STAT (for example, for one of the health centers servicing a smaller population in our study, the per patient cost of using the i-STAT test was $\$ 76.35$, whereas for one of the larger centers the per patient cost was \$31.58).

Finally, some of the sample sizes used in estimating the probabilities for the models were small and could, therefore, reduce the power of our analyses. Future research should consider replicating this analysis in bigger samples. 
While the focus of this study was to examine the clinical and cost effectiveness of POCT for acute presentations, it also highlighted a major cultural benefit of POCT. The prevention of unnecessary medical retrievals enabled many patients to remain in their community, rather than having to suffer the social and emotional trauma associated with dislocation from their families by having to undergo an evacuation for further investigation to a tertiary institution.

\section{Conclusion}

Until now, the current literature from metropolitan tertiary settings indicated that, while POCT can generate improved clinical outcomes, it is generally more expensive than traditional laboratory pathology testing. However, little information is available on the economic effectiveness of POCT in remote settings, where POCT has a particular niche in providing access to critical pathology results in a timely manner. This study demonstrates that POCT can deliver significant cost savings (of the order of nearly AUD \$22 million per annum) for the health care system through ruling out unnecessary and expensive emergency medical retrievals in remote Australia.

\section{Availability of data and materials}

The datasets used and/or analyzed during the current study are available from the corresponding author on a reasonable request.

\section{Ethics approval and consent to participate}

Ethical approval was given by the Menzies School of Health Research Ethics Committee. Reference Number 2015-2469. Patient consent was not required by the ethics committee as only retrospective patient information was sourced and no patient was individually identified in this study.

\section{Acknowledgments}

We thank and acknowledge all health professional staff working at the remote health services for their valuable contribution to improving the health outcomes of remotely located patients. We also thank all past and present members of the Northern Territory Point-of-Care Testing Program Management Committee for their hard work and contribution to the Program's success. This study was funded by a grant awarded by the Emergency Medicine Foundation Ltd.

\section{Author contributions}

BAS initiated and led the design of the study with input from MDSS, BK, and RO. BK performed the economic analysis.
MDSS provided oversight for the study. RO provided expert clinical advice. BAS and BK took primary responsibility for writing the manuscript. MDSS and RO contributed to editing the manuscript. All the authors approved the final manuscript. All authors contributed toward data analysis, drafting and critically revising the paper, gave final approval of the version to be published, and agree to be accountable for all aspects of the work.

\section{Disclosure}

The authors report no conflicts of interest in this work.

\section{References}

1. Australian Institute of Health and Welfare. Indigenous Identification in Hospital Separations Data - Quality Report. cat. no. AIHW 90. Canberra: Australian Institute of Health and Welfare; 2013.

2. Australian Institute of Health and Welfare. Australian Hospital Statistics 2008-2009. Health services no. 34. HSE 84. Canberra: Australian Institute of Health and Welfare; 2010.

3. Northern Territory Department of Health. Fees and Charges Manual 2014. Darwin: Northern Territory Department of Health; 2014.

4. O'Connor TM, Hanks HA, Elcock MS, Turner RC, Veitch C. The medical and retrieval costs of road crashes in rural and remote northern Queensland, 2004-2007: finding from the Rural and Remote Road Safety Study. Med J Aust. 2009;190(2):54-56.

5. CareFlight [webpage on the Internet]. CareFlight Annual Report 2015. Available from: http://careflight.org/_pdf/annual_report_2015. Accessed September 3, 2015.

6. Shephard M. Point-of-care testing comes of age in Australia. Aust Prescriber. 2010;3(1):6-9.

7. Shephard MDS, Causer L, Guy R. Point-of-care testing in rural, remote and Indigenous settings. In: Shephard M, editor. A Practical Guide to Global Point-of-Care Testing. Melbourne, Australia: CSIRO Publishing, Melbourne; 2016:343-354.

8. Spaeth BA, Shephard MD, Schatz S. Point-of-care testing for haemoglobin A1c in remote Australian Indigenous communities improves timeliness of diabetes care. Rural Remote Health. 2014;14(4):2849.

9. St John A, Price CP. Economic evidence and point-of-care testing. Clin Biochem Rev. 2013;34(2):61-74.

10. Quinn AD, Dixon D, Meenan BJ. Barriers to hospital-based clinical adoption of point-of-care testing (POCT): a systematic narrative review, Crit Rev Clin Lab Sci. 2016;53(1):1-12.

11. Laurence CO, Moss JR, Briggs NE, Beilby JJ; PoCT Trial Management Group. The cost-effectiveness of point of care testing in a general practice setting: results from a randomised controlled trial. BMC Health Serv Res. 2010;10:165.

12. Blattner K, Nixon G, Dovey S, Jaye C, Wigglesworth J. Changes in clinical practice and patient disposition following the introduction of point-of-care testing in a rural hospital. Health Policy, 2010;96(1):7-12.

13. Shephard M, Spaeth B, Mazzachi BC, et al. Design, implementation and initial assessment of the Northern Territory Point-of-Care Testing Program. Aust J Rural Health. 2012;20(1):16-21.

14. Shephard M, Spaeth B, Mazzachi B, et al. Toward sustainable pointof-care testing in remote Australia - the Northern Territory i-STAT Point-of-Care Testing Program. Point Care. 2014;13(1):6-11.

15. Spaeth BA, Shephard MD, Omond R. Clinical application of point-ofcare testing in the remote primary health care setting. Qual Prim Care. 2017;25(3):164-175.

16. Spaeth B, Shephard MDS, Auld M, et al. Immediate pathology results now available for all remote Northern Territorians. 14th National Rural Health Conference; March 26-29, 2017; Cairns, Queensland.

17. Fenwick E, Claxton K, Sculpher M. Representing uncertainty: the role of cost-effectiveness acceptability curves. Health Econ. 2001;10(8):779-787. 
18. Philips Z, Ginnelly L, Sculpher M, et al. Review of guidelines for good practice in decision-analytic modelling in health technology assessment. Health Technol Assess. 2004;8(36):1-158.

19. Barton P, Bryan S, Robinson S. Modelling in the economic evaluation of health care: selecting the appropriate approach. J Health Serv Res Policy. 2004;9(2):110-118.

20. Drummond M. Methods for the Economic Evaluation of Health Care Programmes. Oxford: Oxford University Press; 2005.

21. Australian Bureau of Statistics. Census Community Profiles Search, Canberra; 2011. Available from: http://www.abs.gov.au/websitedbs/censushome.nsf/home/communityprofiles?opendocument\&navpos=230. Accessed July 15, 2016.
22. Howick J, Cals JW, Jones C, et al. Current and future use of point-ofcare tests in primary care: an international survey in Australia, Belgium, The Netherlands, the UK and the USA. BMJ Open. 2014;4(8): e005611.

23. Centre for International Economics. The Economic Value of Pathology: Achieving Better Health, and a Better Use of Health Resources: Prepared for Pathology Awareness Australia. Canberra: The Centre for International Economics; 2016.
ClinicoEconomics and Outcomes Research

\section{Publish your work in this journal}

ClinicoEconomics and Outcomes Research is an international, peerreviewed open-access journal focusing on health technology assessment, pharmacoeconomics and outcomes research in the areas of diagnosis, medical devices, and clinical, surgical and pharmacological intervention. The economic impact of health policy and health systems
Submit your manuscript here: https://www.dovepress.com/clinicoeconomics-and-outcomes-research-journal

\section{Dovepress}

organization also constitute important areas of coverage. The manuscript management system is completely online and includes a very quick and fair peer-review system, which is all easy to use. Visit http://www.dovepress.com/testimonials.php to read real quotes from published authors. 